POINTS OF VIEW

\section{Neural circuit diagrams}

\author{
Use alignment and consistency to untangle complex \\ circuit diagrams.
}

Neural circuit diagrams show connections between neurons or brain regions. They are similar to pathways ${ }^{1}$ but typically have more complex connections and more variables, and they can be generated at different scales. Unfortunately, diagrams of even simple circuits are often unnecessarily complex, making understanding brain connectivity maps difficult.

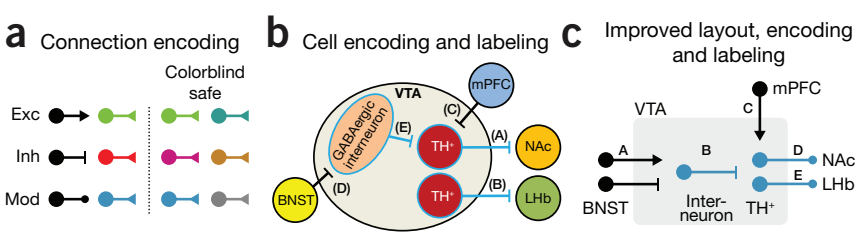

Figure 1 | Use color and shape consistently to encode the type of connection and cell. (a) Use line caps or colors, but not both, for excitory (exc), inhibitory (inh) and neuromodulatory (mod) connections. Use colorblind-safe colors ${ }^{3}$. (b) A busy circuit diagram challenged by tight spacing and angled elements. Figure adapted with permission from ref. 4. (c) Applying consistent size, spacing and alignment of elements without rotation allows for the inclusion of additional relevant information, such as two populations of BNST neurons.

A neural circuit is a network in which brain regions or individual neurons are the nodes and axonal connections are represented by directed edges. Edges can encode many variables but minimally designate the type of neurotransmitter, which determines whether a cell will excite, inhibit or modulate its targets. We recommend limiting this encoding to line caps (Fig. 1a). Variables such as cell type, cell location (layer or brain region), cell morphology, the location of a synapse on a cell, the composition of neurotransmitter receptors on a cell and the strength or density of connections can be encoded using a combination of labels, node position, color and shape, carefully selected on the basis of their salience (Fig. 1b, c).

Encoding several variables without sacrificing information, while still maintaining clarity, is a challenge. To do this, exclude extraneous variables-vary a graphical element only if it encodes something relevant ${ }^{2}$, and do not encode any variables twice.
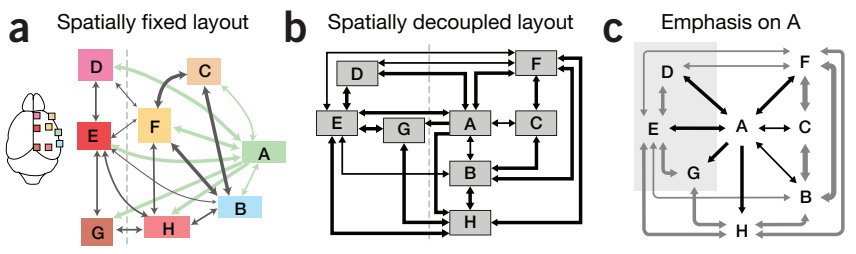

Figure 2 Simplifying node arrangement and discarding unnecessary variables creates clear circuit diagrams without loss of information.

(a) Placing nodes in a neural circuit anatomically makes routing edges difficult and patterns hard to spot. Adapted from ref. 5 with permission from Elsevier. (b) Same circuit with fewer edge crossings and without redundant color. (c) Same circuit with emphasis on node A. Curved edges assist eye movement.
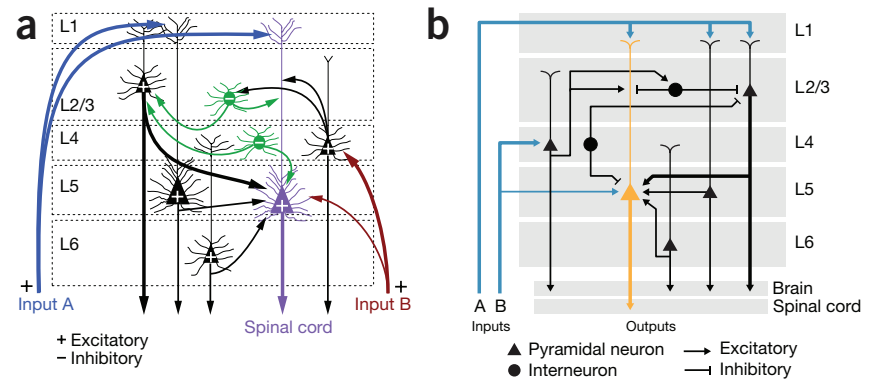

Figure 3 | Maintenance of brain region topology is necessary for some circuit diagrams. (a) Hypothetical cortical microcircuit depicted with common diagram errors: too much morphological detail, inaccurate or ambiguous edge paths, unnecessary variables and weak neurotransmitter encoding. (b) Clarified circuit diagram with emphasis on the two different output regions.

We can apply strategies used to draw pathways ${ }^{1}$ to clarify the flow of information and highlight important features in circuits. If the physical locations of nodes are not important, nodes should be rearranged to clarify the circuit structure and limit the number of edge crossings. In Figure 2a, the position, color and labels all redundantly distinguish brain regions. By removing this redundancy and improving the layout, we make room for additional features to highlight circuit elements (Fig. 2b,c). Other strategies are shown in Supplementary Figure 1.

For neural circuits such as the brainstem auditory circuits, physical arrangement is a fundamental part of function. Another topology that is commonly necessary in neural circuit diagrams is the laminar organization of the cerebral cortex (Fig. 3). When some parts of a circuit diagram are anatomically correct, readers may assume all aspects of the figure are similarly correct. For example, if cells are in their appropriate layers, one may assume that the path that one axon travels to reach another cell is also accurate. Be careful not to portray misleading information-draw edges clearly within or between layers, and always clearly communicate any uncertainty in the circuit.

In some circuits, the location where an axon contacts a postsynaptic neuron can be important to circuit function. A synapse on a cell body may be different than a synapse on an apical dendrite, requiring some cell morphology to be encoded in the circuit diagram. However, to keep the diagram as clear as possible, do not show more morphological information than necessary. Just because the apical dendrite must be shown does not mean all dendrites should be included (Fig. 3). Likewise, just because cell bodies are drawn in the diagram does not mean that the size and shape differences across cells are necessary. The goal is to convey a circuit as clearly and accurately as possible; extraneous variables, such as cell size, will unnecessarily complicate the presentation of your circuit.

Note: Any Supplementary Information and Source Data files are available in the online version of the paper (http://dx.doi.org/10.1038/nmeth.3777).

\section{COMPETING FINANCIAL INTERESTS}

The authors declare no competing financial interests.

\section{Barbara Jeanine Hunnicutt \& Martin Krzywinski}

1. Hunnicutt, B.J. \& Krzywinski, M. Nat. Methods 13, 5 (2016).

2. Wong, B. Nat. Methods 8, 611 (2011).

3. Wong, B. Nat. Methods 8, 441 (2011).

4. Cook, J.B. J. Neurosci. 34, 5824-5834 (2014).

5. Zingg, B. et al. Cell 156, 1096-1111 (2014).

Barbara Jeanine Hunnicutt is a freelance data scientist. Martin Krzywinski is a staff scientist at Canada's Michael Smith Genome Sciences Centre. 